\title{
Nonlinear dynamics and synchronization of computational cognitive model in educational science
}

\author{
Ekemini T. Akpan ${ }^{\mathrm{a}, *}$, Enobong E. Joshua ${ }^{\mathrm{b}}$, Ignatius E. Uduk ${ }^{\mathrm{c}}$ \\ ${ }^{a}$ Department of Science Education, Mathematics Unit, Faculty of Education, University of Uyo, Uyo. \\ ${ }^{b}$ Department of Mathematics, Faculty of Science, University of Uyo, Uyo. \\ ${ }^{c}$ Department of Human Kinetics, Faculty of Education, University of Uyo, Uyo.
}

\begin{abstract}
A computational cognitive model is derived from nonlinear interactions of (Neo) Piagetian-Vygostkian constructs to explain, and predict cognitive processes during collaborative learning. Learning is re-conceptualized as continuous perturbations of cognitive state which unfolds stable cognitive trajectories near Piagetian equilibrium. The model explicates topologically equivalent cognitive patterns, attributed to multi-modal representation of sensory information presented to the learners. Synchronization of the cognitive model is obtained via active control functions which predicts convergence of cognitive states. The synchronized cognitive model is stabilized using Lyapunov matrix equation. These qualitative behaviors emerged due to learner-to-learner and instructor-to-learner scaffolding driven by cognitive executive functions. The dynamical behaviors of the cognitive model are simulated using control parameters with estimated datasets showing viable cognitive trajectories.
\end{abstract}

Keywords: Cognitive model, equilibrium, learning, stability, synchronization, Lyapunov matrix equation.

2020 MSC: 37C75, 91E10, 97C30, 97M10.

(C)2021 All rights reserved.

\section{Introduction}

Recent studies in Mathematical Psychology shows that Neo-Vygostkian and Neo-Piagetian constructs are requisite verbalized theories that link science education research and complex nonlinear dynamical systems [28, 31,34]. Vygotskian constructs explain the theoretical and conceptual framework of collaborative learning. Collaborative learning by nature, creates opportunities for learners to develop their cognition; thinking, reasoning, problem solving, and learning by active communication with more capable peers (instructors) [15]. Also, Piagetian cognitive processes which focused on learners' construction of their knowledge is intertwined with nonlinear dynamic behaviors; learners' cognitive evolution over nested time scale $[19,29]$. Transient cognitive dynamics and cusp catastrophe are robust qualitative behaviors in Piagetian stage-wise cognitive development [21]. In [23], Piagetian core constructs; assimilation and accommodation processes are being modeled as independent control parameters that determine abrupt

\footnotetext{
*Corresponding author

Email addresses: ekeminitakpan@uniuyo.edu.ng (Ekemini T. Akpan), enobongjoshua@uniuyo.edu.ng (Enobong E. Joshua), ignatiusuduk@uniuyo.edu.ng (Ignatius E. Uduk)
}

doi: $10.22436 /$ jnsa.014.01.03

Received: 2020-02-19 Revised: 2020-04-30 Accepted: 2020-05-11 
shift between cognitive developmental stages. The Piagetian notion of equilibration and equilibrium of nonlinear system are equivalent [24]. On one hand, the former is a dynamic process of moving between cognitive state of disequilibrium to equilibrium as learners assimilate new constructs and accommodate problem solving schemes. On the other hand, both can be expressed mathematically by setting the first derivatives of the dynamic equations to zero within a dissipative system [24, 28]. These could be modeled as systems of mathematical equations which incorporate the so-called nonlinear interactions between the cognitive state variables and control parameters $[5,20]$.

\section{Logistic dynamics of cognitive model}

The simplest model applied to cognitive processes in Education is the so-called logistic map which shows nonlinear complexities [16, 17]. This is an iterative scheme of difference equation, which reconceptualize learning as qualitative changes in the learner's cognitive architecture or executive functions over nested time interval, controlled by psychometric parameters,

$$
x_{n+1}=x_{n}+\alpha x_{n}\left(1-\beta x_{n}\right) .
$$

Considering equation (2.1), $x_{\mathrm{n}}$ represents learners' cognitive state at one time period $t>0$, and $x_{\mathrm{n}+1}$ represents cognitive state at the next moment $t+1$. Here $\alpha>0$ denotes neo-Piagetian constructs such as intrinsic learning rate [16]. The parameter $\beta>0$ denotes extraneous cognitive load acting as inhibitory factor during cognitive adaptation processes [25]. The model assumes that mental processing capacity; $\kappa$-capacity is inversely proportional to the extraneous cognitive load parameter, $\beta$. Thus, the independent control parameter $\alpha$ generates complexities in learners' cognitive processes for some variational values [10]. These notions could modify the logistic map in equation (2.1) as follows;

$$
x_{n+1}=x_{n}+\alpha x_{n}\left(1-\frac{x_{n}}{\kappa}\right),
$$

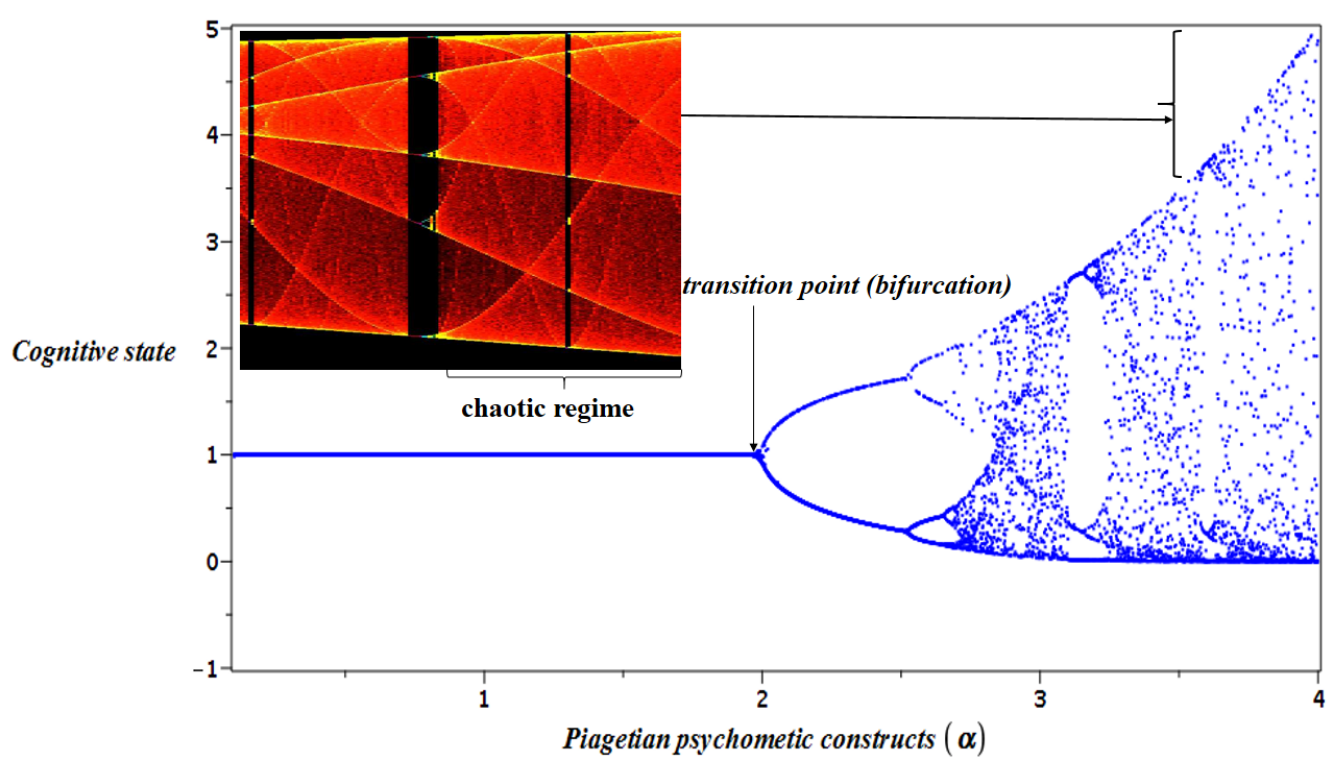

Figure 1: bifurcation diagram of logistic model of learners' stage-wise cognitive behaviors.

with $x_{0}=\eta \in \mathbb{R}^{+}$as the initial chunk of cognitive state. The logistic map as shown in the bifurcation Fig. 1 explains cognitive transitions in Piagetian stage-wise cognitive processes. It connotes learners' cognitive state and behavioural patterns from fixed point attractor to a limit cycle, and from limit cycle into chaos [34]. 


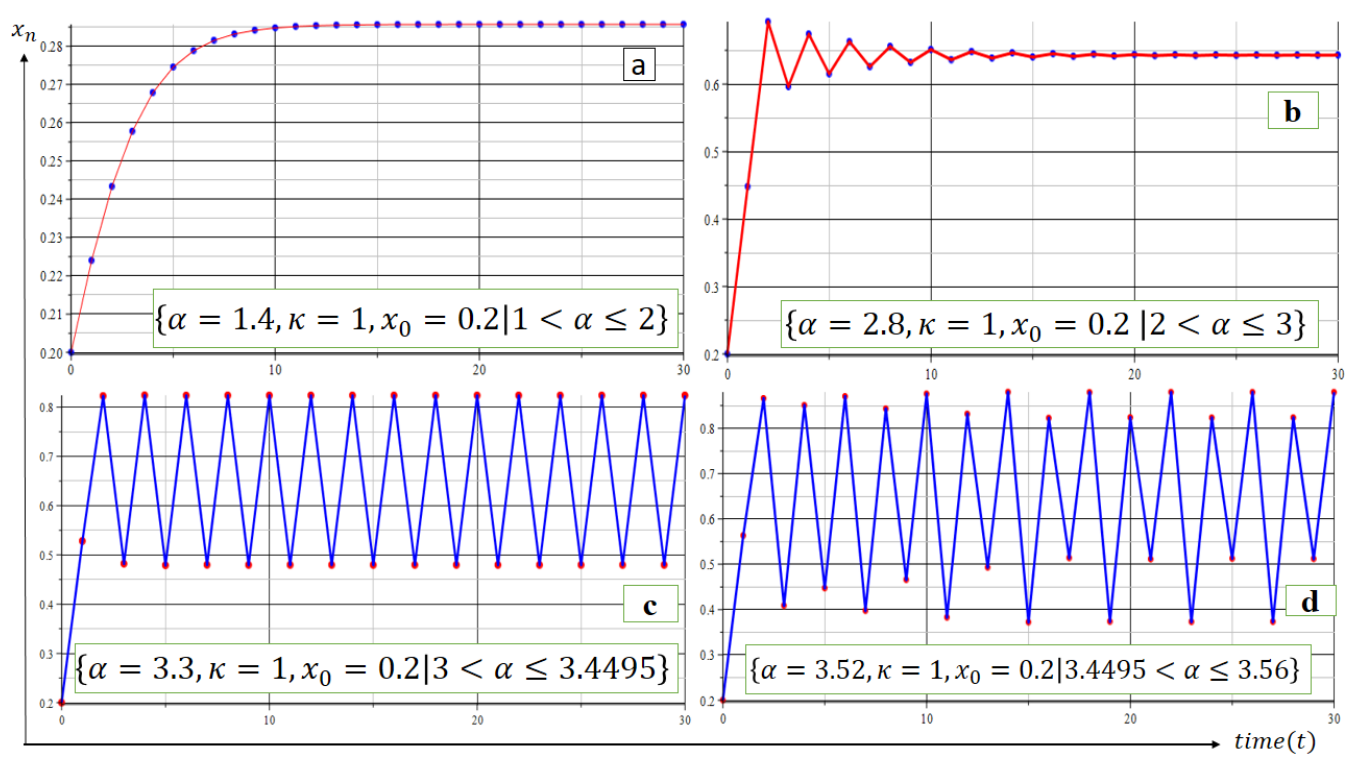

Figure 2: asymptotically stable and cyclic behavior of cognitive state variable over nested time scale.

Fig. 2 shows a time series profile of cognitive state variable plotted against independent control parameter $(\alpha)$, called bifurcation parameter [30]. The cognitive state behavior creates more patterns with further bifurcations from chaotic period to another $[8,34]$. On one hand, the cognitive state behavior is globally asymptotically stable in Fig. 2 (a) and (b) with dynamics that are characteristics of fixed point attractors. On the other hand, the transition point $\alpha=2$ in Fig. 1 starts the bifurcation from period 1 to period 2 where the fixed point attractor becomes a periodic cycle as $\alpha$ increases see Fig. 2 (c) and (d). Towards the end of period 2, where $\alpha$ increases in value, the bifurcation region bifurcates repeatedly, segmenting the profile into four smaller patterns in Fig. 1 and further cycles within cycles emerges as seen in Fig. 3 (a).

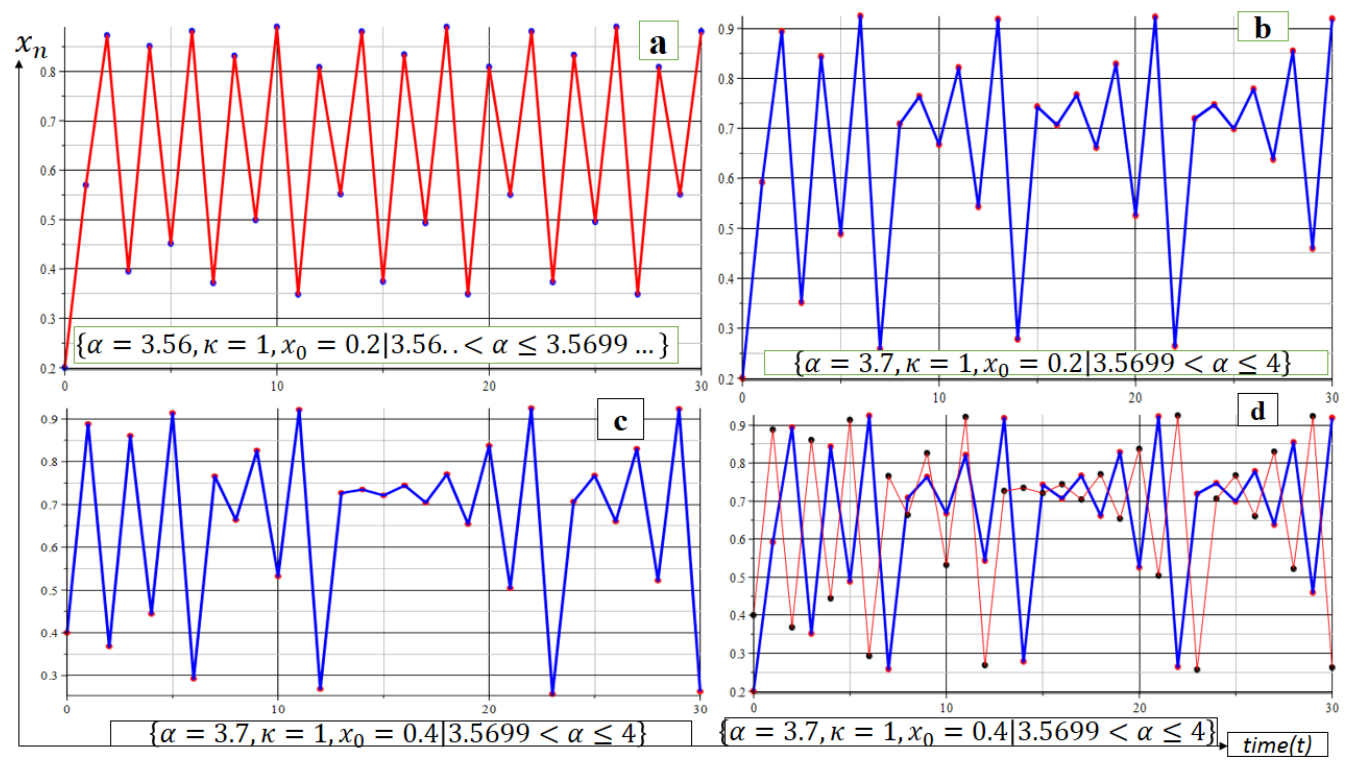

Figure 3: profile of sensitivity of cognitive variable on initial conditions; chaotic behaviors.

Observe that, the system enters period 3, which is full-scale chaos, after one more set of bifurcation Fig. 3 (b). Also, irregular fluctuations in the model Fig. 3 (c) and (d), depicts sensitivity of learner's cognitive state to initial cognitive schemes. Ideally, these dynamics evolves as learners attempt to assimilate new 
knowledge to previously stable cognitive patterns, and major changes called bifurcations create new cognitive structures [4]. The self-organizing nature of the cognitive processes facilitates the evolution of increasing higher ordered thinking and reasoning processes that response to the external environment through efficient and effective decision making.

In [26], coupled logistic maps were used to model synchronization of learners' behavioral pattern during social interactions. The coupling of individuals' dynamics is specified in the following difference equations;

$$
\left\{\begin{array}{l}
x_{1}(t+1)=\frac{r_{1} x_{1}(t)\left(1-x_{1}(t)\right)+\alpha r_{2} x_{2}(t)\left(1-x_{2}(t)\right)}{1+\alpha} \\
x_{2}(t+1)=\frac{r_{2} x_{2}(t)\left(1-x_{2}(t)\right)+\alpha r_{1} x_{1}(t)\left(1-x_{1}(t)\right)}{1+\alpha}
\end{array}\right.
$$

where the dynamical variables $x_{\mathfrak{i}}(\mathfrak{i}=1,2)$ can be interpreted as intensity of cognitive behaviors, and the control parameters, $r_{i}(i=1,2)$ correspond to internal state (e.g., learning rate, learning style, or any other psychometric constructs) that shapes the learners' cognitive behaviors over time, and $\alpha$ is the synchronization parameter representing the strength of coupling or degree of mutual influence during social interactions. The results shown that at each level of coupling, synchronization increases for greater similarity in learners internal state. Also, at each level of similarity in internal state, synchronization increases with strong degree of coupling. Strong coupling promotes full synchronization for learners with different internal state, which in turn guarantees convergence of their cognitive behaviors. If the coupling were to be reduced, their dynamics would immediately diverge. Evidently, learners develop stable cognitive state through synchronization during real-time classroom engagement $[1,3,7,27]$.

\section{Mathematical formulation and parameterization of the cognitive model}

The present section is focused on the assumptions and formulation of a cognitive model to explain, interpret and predict learner-to-instructor cognitive patterns during classroom interaction. This approach is analogous to reinforcement learning theory, and nonlinear dynamical system in education [16, 18]. It is assumed that learners could perform cognitive tasks independently, or with more capable peers and instructors [11]. The stability patterns of learners' cognitive behaviors depend on their internally generated psychometric parameters as well as external stimuli or learning from peers and instructors. Thus, the temporal cognitive model of Kolmogorov-type is given as follows;

$$
\left\{\begin{array}{l}
\dot{x}(t)=\alpha x\left(1-\frac{x}{\xi_{1}}\right)+\eta x z+\mu x y z \\
\dot{y}(t)=\beta y\left(1-\frac{y}{\xi_{2}}\right)+\delta y z+\mu x y z \\
\dot{z}(t)=\rho x z+v y z+\psi(z, \kappa, \sigma) \\
\psi(z)=-\kappa z-\sigma z^{2}
\end{array}\right.
$$

with initial cognitive state defined as $x(0)=\phi_{1}, y(0)=\phi_{2}, z(0)=\phi_{3}$. Observe that learners explore the learning environment with different initial conditions of cognitive states, showing unique level of previous knowledge, and academic histories [32]. These initial conditions may change during teachinglearning processes, with slight changes producing significant learning effects, and vice versa. The system of nonlinear differential equations (3.1) has cognitive variables denoted by $(x(t), y(t), z(t))$ at time $t>0$ as dependent variables, while the Greek letters denote Neo-Piagetian and Neo-Vygotskian control parameters. Here, $\alpha$ and $\beta$ denote different learners' intrinsic learning rates, $\xi_{i}(i=1,2)$ denotes different learners' mental (information) processing capacities. It is assumed that $\xi_{1}$ and $\xi_{2}$ are inversely proportional to their extraneous cognitive loads, respectively. Also, $\eta$ and $\delta$ parameters denote learner-to-learner scaffolding parameters, and $\mu$ represents cooperative proficiency, or coupling strength of mutual influence during social interactions in the classroom [30]. The parameters, $\rho$ and $v$ moderate instructor's resilience 
and adaptation rates as posed by learner's individual differences during teaching-learning process. Also, the function $\psi(z, \kappa, \sigma)$ generates inhibitory effects on the model; it connotes instructors' forgetfulness, or reluctance in response during teaching and learning processes. The cognitive model (3.1) could exhibits complexities, if it satisfies dissipative conditions [6]. Hence, the qualitative properties of such system could be study near their fixed points, attractors, and basin of attractions [13, 22].

\subsection{Local dynamics of the cognitive model}

The cognitive system continuously seeks steady state behavior during classroom interactions. Steady state behavior connotes equilibration processes modified by Piagetian constructs such as accommodation and assimilation; seeking the balance between previous cognitive schemata and incoming stimulus or learning tasks [2, 12]. Define the closed-form of system (3.1) with bounded rationality condition as follows.

$$
\left\{\begin{array}{l}
\dot{X}(t)=F\left(X(t),\left(X(t)=\left((x(t), y(t), z(t))^{\top} \mid(x(t)>0, y(t)>0, z(t)>0)\right)\right.\right. \\
F: \mathbb{R}_{+}^{3} x \mathbb{R}_{+} \rightarrow \mathbb{R}_{+}^{3}, \\
\Omega=\left(X(t) \in \mathbb{R}_{+}^{3} \mid 0 \leqslant x(t) \leqslant \xi_{1}, 0 \leqslant y(t) \leqslant \xi_{2}, 0 \leqslant z(t) \leqslant \frac{\rho \xi_{1}+v \xi_{2}}{\sigma}\right)
\end{array}\right.
$$

The equilibrium state of system (3.2) is defined as

$$
\Sigma=\left(X(0) \mid F\left(X\left(x^{*}, y^{*}, z^{*}\right)\right)=0\right),
$$

where

$$
\begin{aligned}
x^{*} & =\frac{\xi_{1}\left(\mu \sigma z^{* 2}+\kappa \mu z^{*}+\eta v z^{*}+\alpha \kappa\right)}{\mu \rho \xi_{1} z^{*}+\alpha v}, \\
y^{*} & =\frac{\alpha \sigma z^{*}+\alpha \kappa-\eta \xi_{1} z^{*}-\alpha \xi_{1} \rho}{\mu \rho \xi_{1} z^{*}+\alpha v}, \\
P_{1}\left(z^{*}\right) & =A_{3} z^{* 3}+A_{2} z^{* 2}+A_{1} z^{*}+A_{0}=0, \\
A_{3} & =1, A_{2}=\frac{\left(\delta \mu \rho \xi_{1} \xi_{2}+\eta \mu \sigma \xi_{1} \xi_{2}+\kappa \mu^{2} \xi_{1} \xi_{2}\right)}{\mu^{2} \sigma \xi_{1} \xi_{2}}, \\
A_{1} & =\frac{\left(\alpha \mu v \xi_{1} \xi_{2}+\beta \mu \rho \xi_{1} \xi_{2}+\alpha \delta v \xi_{2}+\beta \eta \rho \xi_{1}-\alpha \beta \sigma\right)}{\mu^{2} \sigma \xi_{1} \xi_{2}}, \\
A_{0} & =\frac{\alpha \beta \rho \xi_{1}+\alpha \beta v \xi_{2}-\alpha \beta \kappa}{\mu^{2} \sigma \xi_{1} \xi_{2}} .
\end{aligned}
$$

The cognitive interaction matrix (Jacobian) $J_{i j}(i, j=1,2,3)$ of the model linearized around Piagetian equilibrium $\left(x^{*}, y^{*}, z^{*}\right)$ yields the following matrix

$$
J_{i j}=\left[\begin{array}{ccc}
\frac{\left(\alpha \xi_{1}-2 \alpha x^{*}\right)+\xi_{1}\left(\eta z^{*}+\mu y^{*} x^{*}\right)}{\xi_{1}} & \mu x^{*} y^{*} & \mu x^{*} y^{*}+\eta x^{*} \\
\mu y^{*} z^{*} & \frac{\left(\xi_{2} \beta-2 \beta y^{*}\right)+\left(\delta z^{*}+\mu x^{*} z^{*}\right)}{\xi_{2}} & \mu x^{*} y^{*}+\delta y^{*} \\
\rho z^{*} & v z^{*} & \rho x^{*}-2 \sigma z^{*}-\kappa
\end{array}\right]
$$

with characteristic polynomial

$$
\begin{aligned}
P_{2}(\lambda) & =\lambda^{3}+\operatorname{Trace}\left(J_{i j}\right) \lambda+\left(A_{11}+A_{22}+A_{33}\right) \lambda-\operatorname{det}\left(J_{i j}\right)=0, \\
A_{i j} & =\operatorname{cof}\left(J_{i j}\right), \quad \forall i=j(i, j=1,2,3) .
\end{aligned}
$$


Definition 3.1 ([14]). Let $A \in \mathbb{R}^{n \times n}$ be a real matrix, with $P(\lambda)=\operatorname{det}|\lambda I-A|=a_{0} \lambda^{n}+a_{1} \lambda^{n-1}+\cdots+$ $a_{n-1} \lambda+a_{n}=0$. If $a_{i}>0(i=1,2, \ldots, n)$, then $P(\lambda)$ is Hurwitzian if and only if

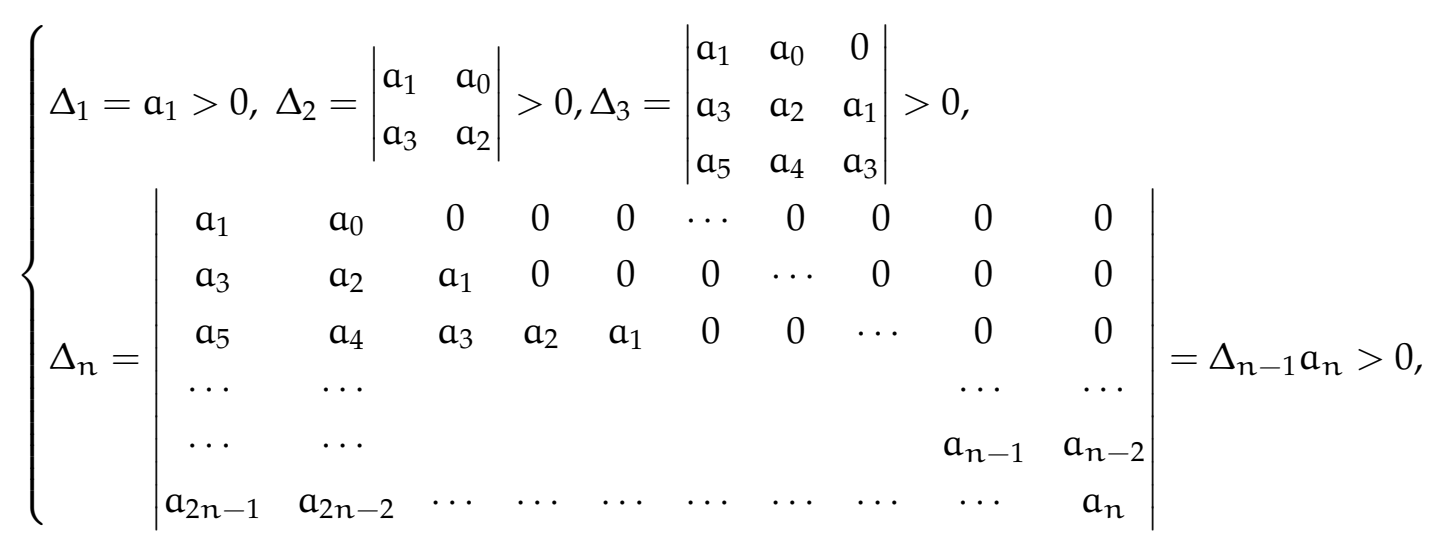

where $a_{s}=0 \forall s<0$ or $s>n$.

Definition 3.2 ([33]). A four tuple dynamical system $\left(T, \mathbb{R}_{+}^{3}, \mathbb{A}, \phi_{*}^{\mathrm{t}}\right)$ is topologically equivalent near an equilibrium point $E^{*}\left(x^{*}, y^{*}, z^{*}\right)$ to a dynamical system $\left(T, \mathbb{R}_{+}^{3}, \mathbb{A}, \phi^{t}\right)$ near an equilibrium point $E\left(x^{*}, y^{*}, z^{*}\right)$ if there exists a homeomorphism $h: \mathbb{R}_{+}^{3} \rightarrow \mathbb{R}_{+}^{3}$ that

1. is defined in a small neighborhood $U \subset \mathbb{R}_{+}^{3}$ of $E^{*}\left(x^{*}, y^{*}, z^{*}\right)$;

2. satisfies $E\left(x^{*}, y^{*}, z^{*}\right)=h\left(E^{*}\left(x^{*}, y^{*}, z^{*}\right)\right)$;

3. maps orbits of the first system in $U$ onto orbits of the second system in $V=F(U) \subset \mathbb{R}_{+}^{3}$, preserving the direction of time.

Remark 3.3. Definition (3.2) provides a way of characterizing two vector fields of the same qualitative dynamics

Lemma 3.4 ([33]). "Consider the sequence of coefficient of real polynomial $P_{n}(\lambda)=a_{n} \lambda^{n}+a_{n-1} \lambda^{n-1}+\cdots+$ $\mathrm{a}_{1} \lambda+\mathrm{a}_{0}$ text defined as $\left\{\mathrm{a}_{\mathrm{n}}\right\}_{i=0}^{i=n}$. Let $\mathrm{k}$ be the total number of sign changes from one coefficient to the next in the sequence, then the number of positive roots of the polynomial is either equal to $k$ or $k-2 m$, where $m$ is an even integer. (Note, if $k=1$, then there is exactly one positive real root)".

Proposition 3.5 ([35]). The cognitive model (system) (3.1) is locally stable and dissipative if

$$
\sum_{i=1}^{3} \lambda_{i}<0,
$$

where $\lambda_{i}$ are eigenvalues of the model (3.1).

Proof. Let us assume that $\mu=0$, the Piagetian equilibrium points of system (3.3) reduces to the following

$$
\left\{\begin{array}{l}
x^{*}=\frac{\xi_{1}\left(-\alpha \delta v \xi_{2}+\beta \eta v \xi_{2}+\alpha \beta \sigma-\beta \eta \kappa\right)}{\alpha \beta \sigma-\alpha \sigma v \xi_{2}-\beta \eta \rho \xi_{1}}, \\
y^{*}=\frac{\xi_{2}\left(\alpha \sigma \rho \xi_{1}-\beta \eta \rho \xi_{1}+\alpha \beta \sigma-\alpha \delta \kappa\right)}{\alpha \beta \sigma-\alpha \sigma v \xi_{2}-\beta \eta \rho \xi_{1}}, \\
z^{*}=\frac{\alpha \beta\left(\rho \xi_{1}+v \xi_{2}-\kappa\right)}{\alpha \beta \sigma-\alpha \sigma v \xi_{2}-\beta \eta \rho \xi_{1}},
\end{array}\right.
$$

and evaluating the cognitive interaction matrix $J_{i j}$ of system (3.4) at the equilibrium points yields the following matrix.

$$
\left[\begin{array}{ccc}
\frac{\alpha\left[\left(\alpha \beta-\delta v \xi_{2}\right)+\eta \beta\left(v \xi_{2}-\kappa\right)\right]}{\alpha\left(\delta v \xi_{1}-\beta \sigma\right)+\beta \eta \rho \xi_{2}} & 0 & \frac{\eta \xi_{1}\left(\alpha \beta \delta-\beta \eta \kappa-\alpha \delta v \xi_{2}+\beta \eta v \xi_{2}\right)}{\alpha \beta \sigma-\alpha \delta v \xi-2-\beta \eta \rho \xi_{1}} \\
0 & \frac{\beta\left[\left(\alpha \beta \sigma+\alpha \delta\left(\rho \xi_{1}-\kappa\right)-\beta \eta \rho \xi_{1}\right)\right]}{\beta \eta \rho \xi_{1}+\alpha\left(\delta v \xi_{1}-\beta \sigma\right)} & \frac{\delta \xi_{2}\left[\beta \eta \rho \xi_{1}-\alpha \rho \xi_{1} \delta+\kappa \delta \alpha-\beta \alpha \sigma\right]}{\beta \eta \rho \xi_{1}+\alpha\left(\delta v \xi_{1}-\beta \sigma\right)} \\
\frac{\alpha \beta \rho\left[\rho \xi_{1}+v \xi_{2}-\kappa\right]}{\alpha \beta \sigma-\alpha \delta v \xi_{2}-\beta \eta \rho \xi_{1}} & \frac{\alpha \beta v\left(\rho \xi_{1}+v \xi_{2}-\kappa\right)}{\beta \eta \rho \xi_{1}+\alpha\left(\delta v \xi_{1}-\beta \sigma\right)} & \frac{\left.\sigma \alpha \beta(\kappa-\rho) \xi_{1}-v \xi_{2}\right)}{\beta \eta \rho \xi_{1}+\alpha\left(\delta v \xi_{1}-\beta \sigma\right)}
\end{array}\right] .
$$


It satisfies the characteristic polynomial,

$$
P_{3}(\lambda)=\lambda^{3}+p_{1} \lambda^{2}+p_{2} \lambda+p_{3}=0,
$$

where

$$
\begin{aligned}
& p_{1}=\frac{\alpha \beta \delta \rho \xi_{1}-\alpha^{2} \delta v \xi_{2}+\alpha \beta \eta v \xi_{2}+\alpha \beta \rho v \xi_{1}+\alpha \beta v \sigma \xi_{2}-\beta^{2} \eta \rho \xi_{1}+\alpha^{2}+\alpha^{2} \beta \sigma+\alpha \beta^{2} \sigma-\alpha \beta \delta \kappa-\alpha \beta \eta \kappa-\alpha \beta \kappa \sigma}{\alpha \beta \sigma-\alpha \delta v \xi_{2}-\beta \eta \rho \xi_{1}}, \\
& p_{2}=\frac{-\alpha \delta^{2} \rho v \xi_{1}^{2} \xi_{2}-\alpha \delta^{2} \rho v^{2} \xi_{1}^{2} \xi_{2}^{2}+\alpha \delta \rho^{2} v \xi_{1}^{2} \xi_{2}+\alpha \delta \eta \rho v^{2} \xi_{1} \xi_{2}^{2}+\beta \delta \eta \rho^{2} v \xi_{1}^{2} \xi_{2}+\beta \delta \rho v^{2} \xi_{1} \xi_{2}^{2}-\beta \eta^{2} \rho^{2} v \xi_{1}^{2} \xi_{2}}{(\alpha \beta)^{-1}\left(\alpha \beta \sigma-\alpha \delta v \xi_{2}-\beta \eta \rho \xi_{1}\right)^{2}} \\
& +\frac{-\alpha^{2} \delta^{2} \rho v \xi_{1} \xi_{2}-\alpha^{2} \delta \rho \sigma v \xi_{1} \xi_{2}-\alpha^{2} \delta \sigma v^{2} \xi_{2}^{2}+2 \alpha \beta \delta \eta \rho \xi_{1} \xi_{2}+\alpha \beta \delta \rho^{2} \sigma \xi_{1}^{2}-\alpha \beta \delta \sigma v^{2} \xi_{2}^{2}-\alpha \beta \eta \rho^{2} \sigma \xi_{1}^{2}}{(\alpha \beta)^{-1}\left(\alpha \beta \sigma-\alpha \delta v \xi_{2}-\beta \eta \rho \xi_{1}\right)^{2}} \\
& +\frac{\alpha \beta \eta \rho \sigma v^{2} \xi_{2}^{2}+2 \alpha \delta^{2} \kappa \rho v \xi_{1} \xi_{2}+\alpha \delta^{2} \kappa v^{2} \xi_{2}^{2}-\alpha \delta \kappa \rho v \xi_{1} \xi_{2}-\beta^{2} \eta^{2} \rho v \xi_{1} \xi_{2}-\beta^{2} \eta \rho^{2} \sigma \xi_{1}^{2}-\beta \eta^{2} \rho v^{2} \xi_{1} \xi_{2}^{2}}{(\alpha \beta)^{-1}\left(\alpha \beta \sigma-\alpha \delta v \xi_{2}-\beta \eta \rho \xi_{1}\right)^{2}} \\
& +\frac{-\beta^{2} \eta \rho \sigma v \xi_{1} \xi_{2}-\beta \delta \eta \kappa \rho v \xi_{1} \xi_{2}+\beta \eta^{2} \kappa \rho^{2} \xi_{1}^{2}+2 \beta \eta^{2} \kappa \rho v \xi_{1} \xi_{2}+\alpha^{2} \beta \delta \rho \sigma \xi_{1}-\alpha^{2} \beta \delta \sigma v \xi_{2}+\alpha^{2} \beta \rho \sigma^{2} \xi_{1}}{(\alpha \beta)^{-1}\left(\alpha \beta \sigma-\alpha \delta v \xi_{2}-\beta \eta \rho \xi_{1}\right)^{2}} \\
& +\frac{\alpha^{2} \beta \sigma^{2} v \xi_{2}+\alpha^{2} \delta^{2} \kappa v \xi_{2}+\alpha^{2} \delta \kappa \sigma v \xi_{2}-\alpha \beta^{2} \eta \rho \sigma \xi_{1}+\alpha \beta^{2} \eta \sigma v \xi_{2}+\alpha \beta^{2} \rho \sigma^{2} \xi_{1}+\alpha \beta^{2} \sigma^{2} v \xi_{2}}{(\alpha \beta)^{-1}\left(\alpha \beta \sigma-\alpha \delta v \xi_{2}-\beta \eta \rho \xi_{1}\right)^{2}} \\
& +\frac{-\alpha \beta \delta \eta \kappa \rho \xi_{1}-\alpha \beta \delta \eta \kappa v \xi_{2}-2 \alpha \beta \delta \kappa \rho \sigma \xi_{1}-2 \alpha \beta \eta \kappa \sigma v \xi_{2}-\alpha \delta^{2} \kappa^{2} v \xi_{2}+\beta^{2} \eta^{2} \kappa \rho \xi_{1}+\beta^{2} \eta \kappa \rho \sigma \xi_{1}}{(\alpha \beta)^{-1}\left(\alpha \beta \sigma-\alpha \delta v \xi_{2}-\beta \eta \rho \xi_{1}\right)^{2}} \\
& +\frac{\alpha^{2} \beta^{2} \sigma^{2}-\beta \eta^{2} \kappa^{2} \rho \xi_{1}-\alpha^{2} \beta \delta \kappa v-\alpha^{2} \beta \kappa \sigma^{2}-\alpha \beta^{2} \eta \kappa \sigma^{2}-\alpha \beta^{2} \kappa \sigma^{2}+\alpha \delta \beta \eta \kappa^{2}+\alpha \beta \delta \kappa^{2} \sigma+\alpha \beta \eta \kappa^{2} \sigma}{(\alpha \beta)^{-1}\left(\alpha \beta \sigma-\alpha \delta v \xi_{2}-\beta \eta \rho \xi_{1}\right)^{2}}, \\
& p_{3}=\frac{2 \alpha \beta \delta \eta \rho v \xi_{1} \xi_{2}-\alpha^{2} \delta^{2} \rho v \xi_{1} \xi_{2}-\beta^{2} \eta^{2} \rho v \xi_{1} \xi_{2}+\alpha^{2} \beta \delta \rho \sigma \xi_{1}-\alpha^{2} \rho \delta \sigma v \xi_{2}-\alpha^{2} \beta \delta \kappa \sigma-\alpha \beta^{2} \eta \kappa \sigma}{\left(\rho \xi_{1}+v \xi_{2}-\kappa\right)^{-1}(\alpha \beta)^{-1}\left(\alpha \beta \sigma-\alpha \delta v \xi_{2}-\beta \eta \rho \xi_{1}\right)^{2}} \\
& +\frac{\alpha^{2} \delta^{2} \kappa v \xi_{2}-\alpha \beta^{2} \eta \rho \sigma \xi_{1}+\alpha \beta^{2}+\alpha \beta^{2} \eta \sigma \nu \xi_{2}-\alpha \beta \delta \eta \rho \kappa \xi_{1}-\alpha \beta \delta \eta \kappa v \xi_{2}+\beta^{2} \eta^{2} \kappa \rho \xi_{1}+\alpha^{2} \beta^{2} \sigma^{2}+\alpha \beta \delta \eta \kappa^{2}}{\left(\rho \xi_{1}+v \xi_{2}-\kappa\right)^{-1}(\alpha \beta)^{-1}\left(\alpha \beta \sigma-\alpha \delta \nu \xi_{2}-\beta \eta \rho \xi_{1}\right)^{2}},
\end{aligned}
$$

whose eigenvalues are required to ensure stability and local dissipativity of the model. Let us consider, the discriminant of equation (3.5) indicated by $\Phi\left(\mathrm{P}_{3}\right)$ as

$$
\Phi\left(P_{3}\right)=-\left|\begin{array}{ccccc}
1 & p_{1} & p_{2} & p_{3} & 0 \\
0 & 1 & p_{1} & p_{2} & p_{3} \\
3 & 2 p_{1} & p_{2} & 0 & 0 \\
0 & 3 & p_{1} & p_{2} & 0 \\
0 & 0 & 3 & 2 p_{1} & p_{2}
\end{array}\right|=18 p_{1} p_{2} p_{3}+\left(p_{1} p_{2}\right)^{2}-4 p_{1}^{3} p_{3}-4 p_{2}^{3}-27 p_{3}^{2}
$$

Using lemma (3.4), the statement of proposition (3.5) is satisfied for $\Phi\left(P_{3}\right)>0, p_{1}>0, p_{2}>0, p_{3}>0$ in (3.6). Similarly, let $\mu \neq 0$, then equation (3.3) gives the required equilibrium points. Suppose the equilibrium points in (3.3) are numerical tractable, and matrix (3.4) is Hurwitzian at the equilibrium points, then the statement of proposition (3.5) is satisfied.

\section{Synchronization dynamics and stability of the cognitive model}

Using definition (3.2), the cognitive system (3.1) has a topologically equivalent system given as follows

$$
\left\{\begin{array}{l}
\frac{d \hat{x}}{d \hat{t}}=\hat{x}-\hat{x}^{2}+\hat{\eta} \hat{x} \hat{z}+\hat{\mu} \hat{x} \hat{y} \hat{z}, \\
\frac{d \hat{y}}{d \hat{t}}=\hat{\beta} \hat{y}-\hat{\beta} \hat{y}^{2}+\hat{\delta} \hat{y} \hat{z}+\hat{z} \hat{x} \hat{y} \hat{z}, \\
\frac{d \hat{t}}{d \hat{x}}=\hat{\rho} \hat{x} \hat{z}+\hat{v} \hat{y} \hat{z}-\hat{k} \hat{z}-\hat{\sigma} \hat{z}^{2},
\end{array}\right.
$$


where $\hat{\eta}=\frac{\eta}{\alpha \sigma}, \hat{\mu}=\frac{\mu \xi_{2}}{\alpha \sigma}, \hat{\beta}=\frac{\beta}{\alpha}, \hat{\delta}=\frac{\delta}{\alpha \sigma}, \hat{\xi}=\frac{\mu \xi_{1}}{\alpha \sigma}, \hat{\rho}=\frac{\rho \xi_{1}}{\alpha}, \hat{v}=\frac{v \xi_{2}}{\alpha}, \hat{\kappa}=\frac{\kappa}{\alpha}, \hat{\sigma}=\frac{1}{\alpha}, \hat{t}=\alpha t, x(t)=$ $\frac{\hat{x}(\hat{t})}{\xi_{1}}, y(t)=\frac{\hat{y}(t)}{\xi_{2}}, z(t)=\alpha \hat{z}(\hat{t})$ via non-dimensionalization. An associative system (slave) of the master equations (4.1) yields

$$
\left\{\begin{array}{l}
\frac{d \hat{x}_{1}}{d \hat{t}}=\hat{x}_{1}-\hat{x}_{1}^{2}+\hat{\eta} \hat{x}_{1} \hat{z}_{1}+\hat{\mu} \hat{x}_{1} \hat{y}_{1} \hat{z}_{1}+\psi_{x}(t) \\
\frac{d \hat{y}_{1}}{d \hat{t}}=\hat{\beta} \hat{y}_{1}-\hat{\beta} \hat{y}_{1}^{2}+\hat{\delta} \hat{y}_{1} \hat{z}_{1}+\hat{z} \hat{x}_{1} \hat{y}_{1} \hat{z}_{1}+\psi_{y}(t) \\
\frac{d \hat{z}_{1}}{d \hat{t}}=\hat{\rho} \hat{x}_{1} \hat{z}_{1}+\hat{v} \hat{y}_{1} \hat{z}_{1}-\hat{k} \hat{z}_{1}-\hat{\sigma} \hat{z}_{1}^{2}+\psi_{z}(t)
\end{array}\right.
$$

where $\psi_{x}(t), \psi_{y}(t), \psi_{z}(t)$ are the active control functions to be determined later. Then, the synchronization error approximation of the two subsystems (4.1) and (4.2) yields,

$$
x_{2}=x_{1}-x, y_{2}=y_{1}-y, z_{2}=z_{1}-z,
$$

then, subtracting systems (4.1) and (4.2) for simplicity yields

$$
\left\{\begin{array}{l}
\frac{d x_{2}}{d t}=x_{2} \underbrace{-x_{1}^{2}+x^{2}+\eta x_{1} z_{1}-\eta x y+\mu x_{1} y_{1} z_{1}-\mu x y z}_{\text {nonlinear }}+\underbrace{\Psi_{x}(t)}_{\text {noncommon }}, \\
\frac{d y_{2}}{d t}=\beta y_{2} \underbrace{-\beta y_{1}^{2}+\beta y^{2}+\delta y_{1} z_{1}-\delta y z+\xi x_{1} y_{1} z_{1}-\xi x y z}_{\text {nonlinear }}+\underbrace{\psi_{y}(t)}_{\text {noncommon }}, \\
\frac{d z_{2}}{d t}=-\kappa z_{2}+\underbrace{\rho x_{1} y_{1}-\rho x y+v y_{1} z_{1}-v y z-\sigma z_{1}^{2}+\sigma z^{2}}_{\text {noncommon }}+\underbrace{\Psi_{z}(t)} .
\end{array}\right.
$$

System (4.3) describes the error dynamics and can be considered as a control problem where the system to be controlled is a linear system with active controller input function as $\left(\Psi(t)=\left(\psi_{x}(t), \psi_{y}(t), \psi_{z}(t)\right)^{\top}\right.$. Suppose the matrix equation of the feedback control system defined as

$$
\left(\psi_{x}, \psi_{y}, \psi_{z}\right)^{\top}=A\left(x_{2}, y_{2}, z_{2}\right)^{\top}
$$

where

$$
A=\left(\begin{array}{ccc}
\frac{\left(\alpha \xi_{1}-2 \alpha x^{*}\right)+\xi_{1}\left(\eta z^{*}+\mu y^{*} x^{*}\right)}{\xi_{1}} & \mu x^{*} y^{*} & \mu x^{*} y^{*}+\eta x^{*} \\
\mu y^{*} z^{*} & \frac{\left(\xi_{2} \beta-2 \beta y^{*}\right)+\left(\delta z^{*}+\mu x^{*} z^{*}\right)}{\xi_{2}} & \mu x^{*} y^{*}+\delta y^{*} \\
\rho z^{*} & v z^{*} & \rho x^{*}-2 \sigma z^{*}-\kappa
\end{array}\right)
$$

is Hurwitzian, then the error dynamic is stabilized for

$$
\left(\dot{x}_{2}(t), \dot{y}_{2}(t), \dot{z}_{2}(t)\right)^{\top}=(A+B)\left(x_{2}(t), y_{2}(t), z_{2}(t)\right)^{\top}
$$

and

$$
B=\left(\begin{array}{ccc}
1 & 0 & 0 \\
0 & \beta & 0 \\
0 & 0 & -\kappa
\end{array}\right),
$$

then, denote (4.5) as

$$
\left(\dot{x}_{2}(t), \dot{y}_{2}(t), \dot{z}_{2}(t)\right)^{\top}=C\left(x_{2}(t), y_{2}(t), z_{2}(t)\right)^{\top} \text { for } C=A+B .
$$

Hence, synchronization of the cognitive system (4.1) with its associated subsystem (4.2) is achieved. The following propositions give sufficient conditions for the system (4.1), and (4.2) to be asymptotically, and globally synchronized for all initial conditions. 
Proposition 4.1. If there exists the feedback gain matrix $\mathrm{B}$ such that the eigenvalues of the matrix $\mathrm{C}=\mathrm{A}+\mathrm{B}$ are negative real or complex with negative real parts, then the dynamical system (4.1) asymptotically synchronizes system (4.2) for all initial conditions.

Remark 4.2. The proof of the theorem is direct. Assuming that the parameters of the cognitive system (4.1) and its associative systems (4.2) are known. The synchronization can be achieved by selecting an active control function (4.4) such that the eigenvalues of the matrix $C$ are negative or complex with negative real parts.

On the other hand, a global synchronization dynamics of the cognitive systems could be achieved using Lyapunov matrix equation defined for positive definite matrix $Q=Q^{\top}$, and a positive definite symmetric matrix $P$ such that,

$$
C^{\top} \mathrm{P}+\mathrm{PC}=-\mathrm{Q}
$$

The direct and explicit solution of the algebraic Lyapunov matrix equation (4.7) involves using CayleyHamilton approach to calculate the matrix exponential function $e^{\mathrm{C}^{\top} t}$ which satisfies the closed form solution of the matrix $\mathrm{P}$ defined as

$$
\mathrm{P}=\int_{0}^{\infty} e^{\mathrm{C}^{\mathrm{T}} \mathrm{t}} \mathrm{Q} e^{\mathrm{Ct}} \mathrm{dt}
$$

Proposition 4.3. The synchronized cognitive model (4.6) is globally stabilized in the sense of Lyapunov for all initial conditions if and only if for any matrix $\mathrm{Q}=\mathrm{Q}^{\top} \succ 0$, there exist a unique matrix $\mathrm{P}=\mathrm{P}^{\top} \succ 0$ such that (4.7) is satisfied.

Proof. To show sufficiency, assume that the synchronized cognitive model (4.6) is time-invariant and proposition (4.1) is satisfied. Let $\mathrm{Q}$ be a positive definite matrix such that $\mathrm{Q}=\mathrm{Q}^{\top}$, and equation (4.8) is well defined. Moreover the integral

$$
\begin{aligned}
P & =\lim _{T \rightarrow \infty} \int_{t=0}^{T} e^{C^{T} t} Q e^{C t} d t \\
& \leqslant\left\|\int_{t=0}^{T} e^{C^{T} t} Q e^{C t} d t\right\| \\
& \leqslant \int_{t=0}^{T}\left\|e^{C^{T} t} Q e^{C t}\right\| \\
& \leqslant\|Q\| \int_{t=0}^{T}\left\|e^{C t}\right\|^{2} d t \\
& \leqslant\|Q\| \int_{t=0}^{T} e^{2 \lambda_{\max }(C) t} d t \\
& \leqslant\|Q\| \int_{t=0}^{T} e^{2 \alpha t} d t \quad\|Q\| \int_{t=0}^{T} e^{-2|\alpha| t} d t=\frac{1}{2|\alpha|} \quad\|Q\| \leqslant \infty,
\end{aligned}
$$

where $\lambda_{\max }(C) \leqslant-\min \Re e\left|\lambda_{i}\right|=\alpha \leqslant 0$. So $P<\infty$ as $T \rightarrow \infty$. Hence, there exists a quadratic Lyapunov functional (positive definite), $V(X(t))=\left(x_{1}(t), y_{2}(t), z_{3}(t)\right)$, defined to ensure the boundedness of the matrix function in (4.8), say,

$$
\mathrm{V}(\mathrm{X}(\mathrm{t}))=\mathrm{X}^{\top}(\mathrm{t}) \mathrm{PX}(\mathrm{t}) \text { for some } \mathrm{P}=\mathrm{P}^{\top} \succ 0 .
$$

Taking the time derivative of (4.9) along the solution trajectories of system (4.6) yields,

$$
\frac{\mathrm{d}}{\mathrm{dt}} \mathrm{V}(\mathrm{X}(\mathrm{t}))=\dot{X}^{\top}(\mathrm{t}) \mathrm{C}^{\top} \mathrm{PX}(\mathrm{t})+\mathrm{X}^{\top}(\mathrm{t}) \mathrm{PC} \dot{X}(\mathrm{t})
$$




$$
\begin{aligned}
& =X^{\top}(t)\left(C^{\top} P+P C\right) X(t) \\
& \leqslant-X^{\top}(t)(t) Q X(t) \leqslant-\lambda_{\min }(Q)\|X(t)\|_{3}^{2}, \quad \forall X(t) \in \mathbb{R}_{+}^{3} .
\end{aligned}
$$

By Lyapunov direct method [9], the time derivative of system (4.9) is negative definite. To show necessity, assume that the matrix $C=A+B$ is hurwitzian (see definition (3.1)), then the matrix functional defined in (4.8) is a bounded solution of eqn (4.7). That is

$$
C^{T} P+P C=\int_{0}^{\infty} C^{T} e^{C^{T} t} Q e^{C t} d t+\int_{0}^{\infty} e^{C^{T} t} Q e^{C t} C d t=\int_{0}^{\infty} \frac{d}{d t}\left(e^{C^{T} t} Q e^{C t}\right) d t=-Q
$$

Hence, the solution of the algebraic Lyapunov function exists for the synchronized cognitive model (4.6). To show that $\mathrm{P} \succ 0$, note that

$$
X^{\top}(t) P X(t)=\int_{0}^{\infty} X^{\top}(t) e^{C^{T} t} Q^{T} Q e^{C t} X(t) d t=\int_{0}^{\infty}\left\|Q e^{C t} X(t)\right\|^{2} d t,
$$

which shows that $\mathrm{P}=\mathrm{P}^{\top}$ and $\mathrm{P} \succeq 0$. Now, suppose, ad absurdum, that $\mathrm{P}$ is not positive semi-definite. Then, there exists $X(0) \in \mathbb{R}^{3}, X(t) \neq 0$, such that $X^{\top}(t) P X(T)=0$, which implies that $Q e^{C t} C X(t)=0, \forall t \geqslant 0$. For $t=0$, it follows that $\mathrm{QX}(\mathrm{t})=0$, which implies that $\mathrm{Q}^{\top} \mathrm{QX}(\mathrm{t})=0$, and hence, $X(\mathrm{t})=0$, which is a contradiction. Hence, $\mathrm{P} \succ 0$.

Finally, assume the observability of the pair $(C, Q)$, then there exist a unique solution of the algebraic Lyapunov equation (4.7). Suppose ad absurdum, that there exist two solutions, say, $\mathrm{P}_{1}, \mathrm{P}_{2}$ and using (4.7) yields;

$$
\left\{\begin{array}{l}
C^{\top} P_{1}+P_{1} C=-Q \\
C^{\top} P_{2}+P_{2} C=-Q
\end{array}\right.
$$

subtracting yields,

$$
\begin{aligned}
C^{\top}\left(P_{1}-P_{2}\right)+\left(P_{1}-P_{2}\right) C & =0, \\
e^{C^{T}}\left(C^{\top}\left(P_{1}-P_{2}\right)+\left(P_{1}-P_{2}\right) C\right) e^{C t} & =0, \\
\frac{d}{d t}\left(e^{C^{\top} t}\left(P_{1}-P_{2}\right) e^{C t}\right) & =0 \\
\int_{0}^{\infty} \frac{d}{d t}\left(e^{C^{\top} t}\left(P_{1}-P_{2}\right) e^{C t}\right) & =0, \\
-\left(P_{1}-P_{2}\right) & =0 .
\end{aligned}
$$

This leads to the contradiction $\mathrm{P}_{1}=\mathrm{P}_{2}$, and hence, there exists a unique solution of the algebraic Lyapunov (4.7), and numerically tractable.

\section{Discussion of results and numerical simulations of the cognitive model}

Table 1 shows cognitive variables and parameters with estimated data-sets of two cognitive typologies used to demonstrate stability patterns, and synchronization dynamics of the model.

Figure 4 shows the cognitive trajectories of the first cognitive loop $\left(^{*}\right)$ in Table 1 exhibiting a stable pattern over time in the neighborhood of Piagetian equilibrium point; (1.5320, 2.5884, 0.15744) with cognitive interaction matrix defined as;

$$
\left(\begin{array}{ccc}
-3.4725 & 0.0289 & 0.7056 \\
0.0489 & -7.7651 & 4.3589 \\
0.079 & 0.2047 & -2.4121
\end{array}\right)
$$


Then, its characteristic polynomial

$$
\lambda^{3}+12.17909168 \lambda^{2}+36.64538318 \lambda+22.23627034
$$

has negative spectra $(-0.8107,-3.4749,-7.8935)$ which guarantees local stability and dissipativity of the cognitive model.

Table 1: (Neo)Piagetian-Vygotskian qualitative variables/estimated parameters.

\begin{tabular}{|c|c|c|c|c|}
\hline cognitive state $^{*}$ & cognitive state ${ }^{* *}$ & descrip., of psychometric parameters & values* & values $^{* *}$ \\
\hline$x(t)$ & $x_{2}(\tau)$ & learner's cognitive state at time $\tau, t \geqslant 0$ & variable & variable \\
\hline$z(t)$ & $z_{2}(\tau)$ & instructor's cognitive state at time $\tau, t \geqslant 0$ & variable & variable \\
\hline$z(t)$ & $z_{2}(\tau)$ & instructor's cognitive state at time $\tau, t \geqslant 0$ & variable & variable \\
\hline$x(0)$ & $x_{2}(0)$ & learner's initial cognitive state (1) & 3.0000 & 3.0000 \\
\hline$y(0)$ & $y_{2}(0)$ & learner's initial cognitive state (2) & 2.0000 & 2.0000 \\
\hline$z(0)$ & $z_{2}(0)$ & instructor's initial cognitive state & 7.0000 & 7.0000 \\
\hline$\alpha$ & $\hat{\alpha}$ & learner's intrinsic learning rates(1) & 3.4000 & 1.0000 \\
\hline$\beta$ & $\hat{\beta}$ & learner's intrinsic learning rate (2) & 7.5000 & 2.2059 \\
\hline$\xi_{1}$ & $\hat{\xi_{1}}$ & learner's mental processing capacities(1) & 1.5000 & 1.0000 \\
\hline$\xi_{2}$ & $\hat{\xi_{2}}$ & learner's mental processing capacities (2) & 2.5000 & 1.0000 \\
\hline$\eta$ & $\hat{\eta}$ & instructor to learner scaffolding parameters $(1)$ & 0.1500 & 0.0074 \\
\hline$\delta$ & $\hat{\delta}$ & instructor to learner scaffolding parameters(2) & 1.5000 & 0.0836 \\
\hline$\mu$ & $\hat{\mu}, \hat{\xi}$ & cooperative proficiencies & 0.1200 & 0.0148 \\
\hline & & & & 0.0089 \\
\hline$\rho$ & $\hat{\rho}$ & instructor's adaptation rates (1) & 0.0500 & 0.0220 \\
\hline$v$ & $\hat{v}$ & instructor's adaptation rates (2) & 1.3000 & 0.9559 \\
\hline K & $\hat{K}$ & instructor's forgetting coefficients & 2.5000 & 0.7353 \\
\hline$\sigma$ & $\hat{\sigma}$ & instructor's inhibitory effects & 5.9800 & 0.2941 \\
\hline$x^{*}$ & $\hat{\chi}^{*}$ & learner's Piagetian equilibrium points(1) & 1.5320 & 1.0053 \\
\hline$y^{*}$ & $\hat{y}^{*}$ & learner's Piagetian equilibrium points(2) & 2.5884 & 1.0000 \\
\hline$z^{*}$ & $\hat{z}^{*}$ & instructor's Piagetian equilibrium points & 0.1575 & 0.0011 \\
\hline
\end{tabular}

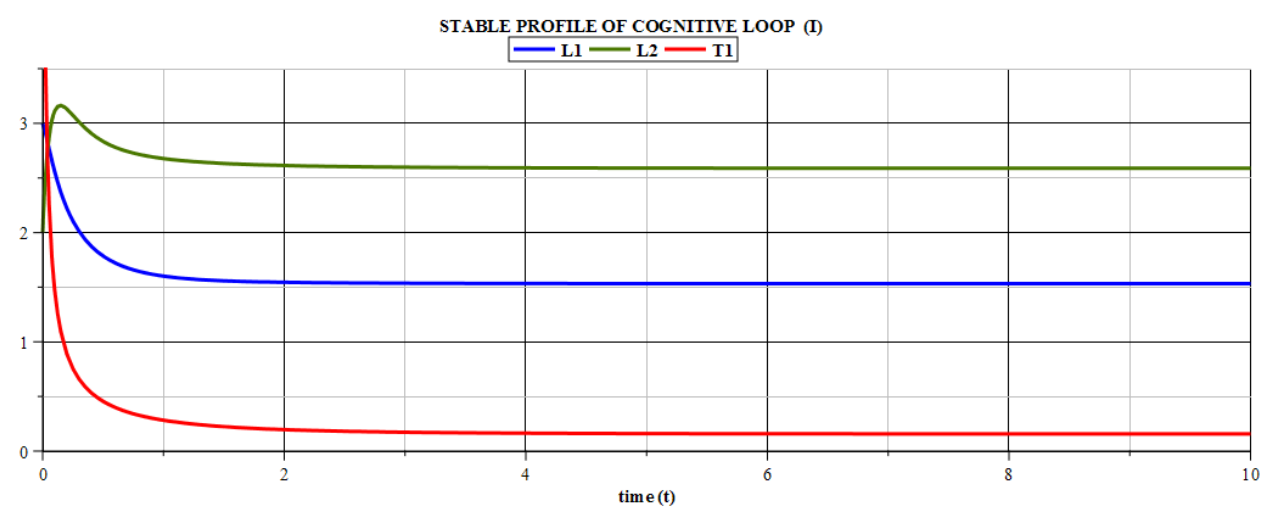

Figure 4: local asymptotic stable cognitive trajectories of learners $\left(\mathrm{L}_{1}, \mathrm{~L}_{2}\right)$-instructor $\left(\mathrm{T}_{1}\right)$ interactions.

Figure 5 shows the cognitive trajectories of the second cognitive loop $\left(^{* *}\right)$ in Table 1 preserving stability pattern over time in the neighborhood of Piagetian equilibrium point; $(1.0019,1.0036,0.087)$ with cognitive interaction matrix defined as follows

$$
\left(\begin{array}{ccc}
-1.0053142 & 0.005334396607 & 5.049645135 \\
9.3660 e-6 & -2.205980241 & 0.0925162324 \\
2.3341 e-5 & 0.001011435484 & -0.02221846803
\end{array}\right) .
$$

Then, its characteristic polynomial

$$
\lambda^{3}+3.233512949 \lambda^{2}+2.2884192 \lambda+0.04891986679
$$


has negative spectra $(-0.02205573817,-1.0054341,-2.20602311)$ which guarantees local stability and dissipativity of the cognitive model.

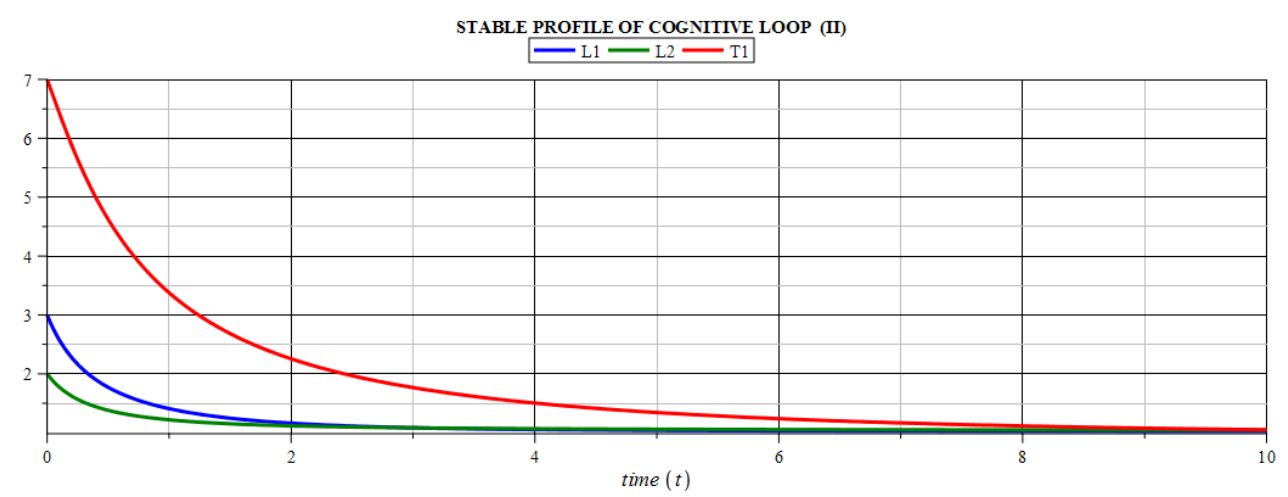

Figure 5: local asymptotic stable cognitive trajectories of learners $\left(\mathrm{L}_{1}, \mathrm{~L}_{2}\right)$-instructor $\left(\mathrm{T}_{1}\right)$ interactions.

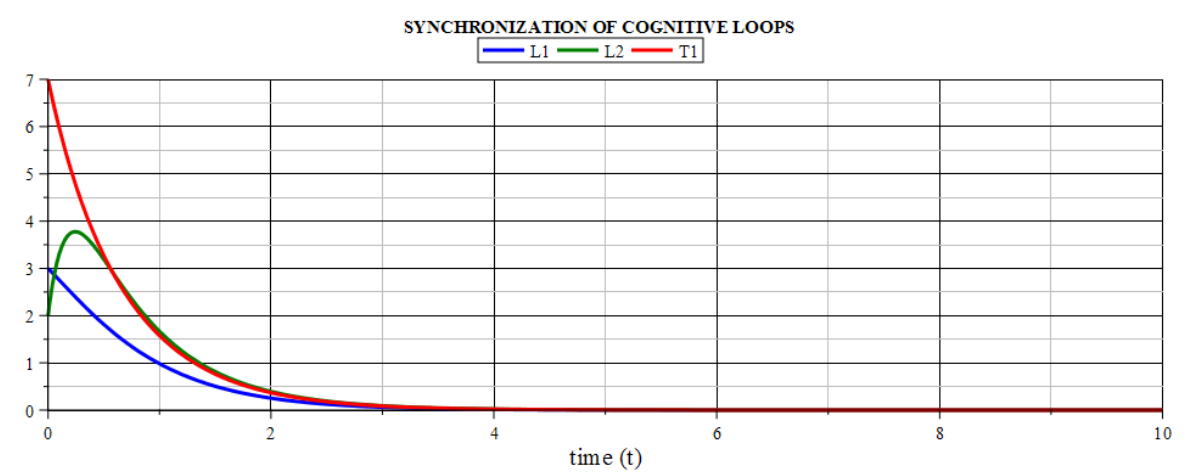

Figure 6: synchronized and global asymptotic stable patterns of learners $\left(\mathrm{L}_{1}, \mathrm{~L}_{2}\right)$ and instructor $\left(\mathrm{T}_{1}\right)$ cognitive interactions.

Figure 6 depicts synchronization dynamics of the two (2) cognitive loops. In one hand, proposition 4.1 is satisfied, because the cognitive interaction matrix (5.1) is Hurwitzian with a feedback gain matrix B defined as follows

$$
B=\left(\begin{array}{ccc}
1 & 0 & 0 \\
0 & 2.2059 & 0 \\
0 & 0 & -0.7353
\end{array}\right)
$$

with synchronized cognitive interaction matrix $C$ defined as

$$
C=\left(\begin{array}{ccc}
-2.4725 & 0.0289 & 0.7056 \\
0.0489 & -5.5592 & 4.3584 \\
0.0078 & 0.2047 & -1.6768
\end{array}\right)
$$

On the other hand, the synchronized dynamics of the cognitive model is globally stable as shown in Figure 6 with Lyapunov matrix P defined as

$$
\mathrm{P}=\left(\begin{array}{ccc}
0.2024 & 2.347 e-3 & 0.0387 \\
2.347 e-3 & 0.0925 & 0.0702 \\
0.0387 & 0.07017 & 0.4969
\end{array}\right)
$$

satisfying the statement of proposition (4.3) for any identity $3 \times 3$ matrix Q. 


\section{Conclusion}

This study provides qualitative behaviors of cognitive processes using the approach of complex nonlinear dynamical system. The notion of Piagetian equilibrium which is analogous to equilibrium of a nonlinear dynamical system, guarantees the existence of fixed point/attractors of the cognitive model. The model is dissipitive, locally stabilized in the neighborhood of Piagetian equilibrium. Its exhibits global synchronization dynamics for different cognitive topologies. The dynamics of the cognitive model were characterized as cognitive trajectories visible via simulations of the cognitive state variables with estimated control parameters. In applications to educational science, a stable cognitive pattern ensures effective and efficient cognitive performance and robustness in decision making. Synchronization dynamics of the model explains rationality of cognitive convergence in problem solution during collaborative learning.

\section{Acknowledgment}

We appreciate the tremendous support of Tertiary Education Trust Fund (TETFUND) for Institution Based Research(IBR) grant (TETFUND/IBR/UNIUYO/2017) of the University of Uyo. We thank Prof. Dimitrios Stamovlasis for his remarks. We acknowledged our anonymous reviewers for their constructive comments.

\section{References}

[1] J. Bhattacharya, Cognitive neuroscience: Synchronizing brain in the classroom, Current Biology, 27 (2017), 339-363. 2

[2] H. B. Bormanaki, The role of equilibration in Piaget's thoery of cognitive development and it implication for receptive skills: A theoretical study, J. Lang. Teach. Res., 8 (2017), 996-1005. 3.1

[3] S. Dikker, L. Wan, I. Davidesco, J. J. Van Barel, M. Ding, D. Poeppel, Brain-to-brain synchrony tracks real-world dynamics group interactions in the classroom, Cuurent Biology, 27 (2017), 1375-1389. 2

[4] C. D. Ennis, Reconceptualizing learning as dynamical system, J. Curriculum Supervision, 7 (1992), 115-130. 2

[5] S. Farrell, S. Lewandowsky, An introduction to cognitive modelling, in: An introduction to model-based cognitive neuroscience, Springer, New York, (2015). 1

[6] D. L. Gistrap, Dissipative structures in educational change: Prigogine and the academic, Int. J. Leader. Educat. Theor. Prac., 10 (2007), 49-69. 3

[7] P. Greenwood, P. Parasuraman, Neuronal and cognitive plasticity: A neurocognitive framework for ameliorating cogntitive aging, Frontiers in Aging Neuroscience, 2 (2010), 14 pages 2

[8] S. J. Guastello, L. S. Liebovich, Introduction to nonlinear dynamics and complexity, In: Chaos and complexity in psychology: The theory of nonlinear dynamical systems, Cambridge University Press, Cambridge, (2009). 2

[9] W. M. Haddad, V. S. Chelloboina, Nonlinear dynamical systems and controls: A Lyapunov-based approach, Princeton University Press, Princeton, (2008). 4

[10] R. A. Heath, Nonlinear dynamics: Techniques and applications in psychology, Mahwah, Erlbaum, (2000). 2

[11] A. Konar, Artificial intelligence and soft computing: Behavioural and cognitive modelling of the human brain, CRC Press, Boca Raton, (2000). 3

[12] M. Koopsman, D. Stamovlasis, Complex dynamical systems in education: Concepts, methods and applications, Springer, Cham, (2016). 3.1

[13] R. Lamb, A. Cavagnetto, A. Akmal, Examination of the nonlinear dynamic systems associated with student cognition while engaging in science information processing, Int. J. Sci. Math. Edu., 14 (2016), 187-205. 3

[14] X. X. Liao, L. Q. Wang, P. Yu, Stability of dynamical systems, Elsevier B. V., Amsterdam, (2014). 3.1

[15] L. Lin, Investigating chinese HE EFL classrooms, Springer, New York, (2005). 1

[16] P. Lockwood, M. Klein-Flügge, Computational modelling of social cognition and behaviour-a reinforcement learning primer, Social cognitive and affective neuroscience, 2020 (2020), 29 pages. 2, 2, 3

[17] N. Milescu, M. V. Rusu, C. Berlic, A nonlinear dynamical system approach of learning, Romanian Reports in Physics, 5 (2013), 1547-1556. 2

[18] B. N. Nicoleiscu, T. C. Petrecu, Dynamical system theory: A powerful tool in educational sciences, Procedia-Social and Behavioral Sciences, 76 (2013), 581-587. 3

[19] J. Piaget, To understand is to invent: The future of education, Free Press, New York, (1973). 1

[20] T. Poston, I. Stewart, Catastrophe theory and its applications, Pitman, London, (1978). 1

[21] M. I. Rabinovich, R. Huerta, P. Varona, V. S. Afraimovich, Transient cognitive dynamics, metastability, abd decision making, Comput. Biol., 4 (2008), 9 pages. 1 
[22] E. Reithmeier, Periodic solutions of nonlinear dynamical systems: Numerical computation, stability, bifurcation and transition to chaos, Springer-Verlag, Berlin, (1991). 3

[23] D. G. Saari, A Qualitative Model for the Dynamics of Cognitive Processes, J. Mathematical Psychology, 15 (1977), 145-168. 1

[24] D. Stamovlasis, Catastrophe theory: Methodology, epistemology, and applications in learning science, in: Complex dynamical systems in education: Concepts, methods and applications, Springer International Publishing, Cham, (2016). 1

[25] J. Sweller, P. Ayres, S. Kalyuga, Cognitive load theory, Springer, New York, (2011). 2

[26] R. R. Vallacher, A. Nowak, The dynamics of human experience: Fundamental of dynamical social psychology, in: Chaos and complexity in psychology: Theory of nonlinear dynamical sytems, Cambridge University Press, New York, (2009).

[27] R. R. Vallacher, A. Nowak, M. Zochowski, Dynamics of social coordination: The synchronization of internal states in close relationship, Interaction Studies, 6 (2005), 35-52. 2

[28] H. L. J. Van der Mass, P. C. Molenaar, Stagewise Cognitive Development: An Applicatiion of Catastrophe Theory, Psychological Review, 99 (1992), 395-417. 2

[29] P. Van Geert, A Dynamic Systems Model of Cognitive and Language Growth, Psychological Review, 98 (1991), 3-53. 1

[30] P. Van Geert, H. Steenbeck, The dynamics of scaffolding, New Ideas in Psychology, 23 (2005), 115-128. 1 2,3

[31] Z. Wang, Z. Liu, C. Zheng, Qualitative analysis and control of complex neural networks with delays, Springer, Berlin, (2016). 1

[32] G. Weichhart, The learning environment as a chaotic and complex adaptive system, Systema: Connecting Matter, Life, Culture and Technology, 1 (2013), 36-53. 3

[33] S. Wiggins, Introduction to applied nonlinear dynamical systems and chaos, Springer-Verlag, New York, (2003). 3.2, 3.4

[34] G. Young, Development and causality: Neo-Piagetian perspectives, Springer, New York, (2011). 1, 2, 2

[35] M. Zak, J. P. Zbilut, R. E. Meyers, From instability to intelligence: Complexity and predictability in nonlinear dynamics, Springer-Verlag, Berlin, (1997). 3.5 